\title{
Planck scale symmetry breaking and majoron physics
}

\author{
I.Z. Rothstein \\ Department of Physics, University of Michigan, Ann Arbor, MI 48109, USA \\ K.S. Babu and D. Seckel \\ Bartol Research Institute, University of Delaware, Newark, DE 19716, USA \\ Received 19 January 1993 \\ Accepted for publication 15 April 1993
}

\begin{abstract}
Majoron models provide neutrino masses via the spontaneous breaking of a global U(1) symmetry. However, it may be argued that all global symmetries will be explicitly violated by gravitational effects. We show that it is possible to preserve most of the usual features of majoron models by invoking $\mathrm{U}(1)_{\mathrm{B}-\mathrm{L}}$ to be a gauge symmetry and adding a second singlet scalar field. The majoron gets a small model dependent mass. The couplings of majorons to neutrinos may be of ordinary strength or may be made arbitrarily weak. We discuss the cosmological and astrophysical consequences of majoron models in the context of a model dependent majoron mass and neutrino coupling. For an appropriate choice of parameters majorons can play the role of dark matter.
\end{abstract}

\section{Introduction}

Majoron models [1,2] were designed to accommodate a wide range of neutrino masses. In most theories, small neutrino masses are achieved via the seesaw mechanism [3]. In grand unified models, seesaw neutrino masses are naturally of order $10^{-2} \mathrm{eV}$ or less, but in majoron models the scale of lepton number violation is left as a free parameter, and so larger neutrino masses are not implausible. Another attractive feature of majoron models is that they contain a spontaneously broken global symmetry, complete with a Nambu-Goldstone boson, i.e., the majoron. This allows for neutrinos with mass greater than $30 \mathrm{eV}$ to escape the constraint from the closure density of the Universe [4], since they may decay into a lighter neutrino and a majoron.

Besides allowing for neutrino mass and neutrino decay, majoron models lead to a host of other cosmological, astrophysical, and laboratory consequences [5]. Since the majoron couples to neutrinos there exist a number of constraints based on possible majoron production in neutrino rich environments; e.g. excess cooling of supernova cores by majoron emission [6] or, a majoron contribution to the energy 
density of the Universe which could destroy the successful predictions of big-bang nucleosynthesis [7]. Couplings to other standard model particles arise through loops, which leads to constraints based on the evolution of red giants [8] and horizontal branch stars. Majoron models also possess global U(1) cosmic strings, although we know of no important consequences of such relics. Finally, there are a number of laboratory experiments which are sensitive to majoron models. The most important examples are the width of the $\mathrm{Z}$ boson as measured at LEP [9], which eliminates the simplest of triplet majoron models [2]; and the absence of three-body neutrinoless double beta decay events [10]. We mention these features now because they illustrate the richness of majoron models, and they are all modified by our attempt to reconcile majoron models with short distance symmetry breaking induced by gravitational effects.

A criticism of majoron models is that the global symmetry is not necessitated by the known particle spectrum. Although there may be nothing wrong with this, it has been argued that quantum gravitational effects should explicitly violate all global symmetries [11]. Assuming this to be true, a simple way to save the majoron models is to ensure that the global symmetry be accidental. This can be accomplished if we gauge $\mathrm{U}(1)_{\mathrm{B}-\mathrm{L}}$ and choose the quantum numbers of the scalar fields to ensure that there are no gauge invariant operators of dimension four or less which break the accidental symmetry. The higher dimensional operators which explicitly break the accidental U(1) will be suppressed by powers of $M_{\mathrm{pl}}$.

A small explicit symmetry breaking has two immediately consequences. First, the majoron will acquire a small mass, which in turn has many astrophysical and cosmological implications. Second, in a cosmological setting, a U(1) symmetry that is broken both spontaneously and explicitly implies the formation of a network of strings and walls. The ability of this network to dissipate places constraints on the details of the model.

In the usual majoron models there is a direct relationship between neutrino mass and the coupling of neutrinos to majorons. In the present context there must be two scalar fields in order to break both the gauge symmetry and the accidental global symmetry. Both must have charge under B - L, but only one may couple to right-handed neutrinos to power the seesaw mechanism. The majoron is predominantly the scalar with the smaller vacuum expectation value (VEV). If the smaller VEV is also associated with neutrino masses, then the linear relationship between neutrino mass and coupling to majorons is preserved; but if the larger VEV is associated with neutrino mass the coupling to majorons will be suppressed by a ratio of small to large VEVs.

This paper gives an in-depth discussion of the issues just summarized. Sect. 2 reviews the singlet majoron model and its phenomenology. Sect. 3 discusses one way to build majoron models containing an automatic symmetry to protect against gravity. Sect. 4 discusses the cosmological constraints that arise from giving the majoron a mass. The possibility that majorons play the role of dark matter is also 
examined in this section. Sect. 5 is a discussion of the model constraints that arise from a consideration of the string-wall network. Sects. 6 and 7 review the constraint from big-bang nucleosynthesis and SN 1987A respectively. The last section contains a brief summary.

Similar cosmological and astrophysical analyses have been performed for axion models with gravitational effects [12,13], and for a broad class of models where the mass of the Goldstone boson is due to instanton effects in an unspecified strongly interacting sector of the theory [14]. The situation for axion models differs from the majoron models presented here due to upper limits on the neutron electric dipole moment, which place severe constraints on the strength of explicit symmetry breaking due to gravitational effects. These translate into severe constraints on the model, e.g. the lowest dimensional operator that is allowed from strong $C P$ considerations is $d=10$. No similar constraint exists for majoron models, and so $d=5$ is viable.

The phenomenology of textures [15] and neutrino masses [16] have also been reexamined in the context of Planck suppressed symmetry breaking. While this work was in preparation we became aware of a paper by Akhmedov et al. [17] which considers some of the same issues as the present paper for majoron physics.

\section{The minimal majoron model}

The simplest majoron model [1] is built by adding $\mathrm{SU}(1)_{\mathrm{L}} \times \mathrm{U}(1)_{\mathrm{Y}}$ singlet neutrinos $\left(N^{\prime}\right)^{\star}$ as well as a singlet scalar $(\phi)$ to the spectrum of the standard model. The singlet scalar couples only to the singlet neutrinos because it carries lepton number $L=-2$. The relevant part of the lagrangian which carries these extra fields is given by

$$
\begin{aligned}
L_{\text {new }}= & \frac{1}{2} \bar{N}_{i}^{\prime} i \not N_{i}^{\prime}+V(H, \phi) \\
& +\left[\frac{1}{4} \lambda_{i j}^{(1)} N_{i}^{\prime}\left(1+\gamma_{5}\right) N_{j}^{\prime} \phi+\frac{1}{4} \lambda_{i j}^{(2)} \bar{\nu}_{i}^{\prime}\left(1+\gamma_{5}\right) N_{j}^{\prime} H+\text { h.c. }\right],
\end{aligned}
$$

where $H$ denotes the standard model Higgs doublet and $i, j$ are generation indices. We use four-component majorana spinors $\left(N^{\mathrm{c}}=N, \nu^{\mathrm{c}}+\nu\right)$, and assume, for simplicity, that the Yukawa coupling matrices $\lambda^{(1)}, \lambda^{(2)}$ contain no $C P$ violating phases. The Higgs potential can be written as

$$
V=\kappa_{1}\left(H H^{\dagger}-v_{H}^{2}\right)^{2}+\kappa_{2}\left(H H^{\dagger}-v_{H}^{2}\right)\left(\phi \phi^{*}-v^{2}\right)+\kappa_{3}\left(\phi \phi^{*}-v^{2}\right)^{2} .
$$

If $4 \kappa_{1} \kappa_{3}>\kappa_{2}^{2}$, then $\langle H\rangle=v_{H}$ and $\langle\phi\rangle=v$. Utilizing the non-linear realization of

* We use primes to denote the interaction basis for the neutrinos and leave off the primes to denote the mass basis. 
the global symmetry, the scalar may be written as

$$
\phi=(\rho+v) \exp \left(i \frac{\chi}{v}\right)
$$

where the majoron will be $\chi$, and the real scalar piece $\rho$ will be massive. We can eliminate the majoron field from the mass matrix by transforming the neutrino fields; $N_{i}^{\prime} \rightarrow \exp \left(-i \chi \gamma_{5} / 2 v\right) N_{i}^{\prime}$ and $\nu_{i}^{\prime} \rightarrow \exp \left(i \chi \gamma_{5} / 2 v\right) v_{i}^{\prime}$; leaving

$$
\begin{gathered}
L=\frac{1}{2} M_{i j} \bar{N}_{i}^{\prime} N_{j}^{\prime}+\frac{1}{2} m_{\mathrm{D}, i j} \bar{\nu}_{i}^{\prime} N_{j}^{\prime}+\frac{1}{2} \bar{N}_{i}^{\prime} i \partial N_{i}^{\prime} \\
+\frac{\partial_{\mu} \chi\left(\bar{\nu}_{i}^{\prime} \gamma^{\mu} \gamma_{5} \nu_{i}^{\prime}-\bar{N}_{i}^{\prime} \gamma^{\mu} \gamma_{5} N_{i}^{\prime}\right)}{4 v},
\end{gathered}
$$

where

$$
m_{\mathrm{D}, i j}=\lambda_{i j}^{(2)} v_{H}, \quad M_{i j}=\lambda_{i j}^{(1)} \iota,
$$

the subscript $\mathrm{D}$ indicates a Dirac mass term coupling $\nu^{\prime}$ and $N^{\prime}$, and we have not written the coupling of neutrinos to $\rho$ particles since we are not interested in theories where the $\rho$ is light. This will yield the following seesaw mass matrix

$$
L_{\text {mass }}=\frac{1}{2}\left(\bar{\nu}^{\prime} \bar{N}^{\prime}\right) M\left(\begin{array}{c}
\nu^{\prime} \\
N^{\prime}
\end{array}\right)
$$

where

$$
M=\left(\begin{array}{cc}
0 & m_{\mathrm{d}, i j} \\
m_{\mathrm{D}, i j}^{\mathbf{T}} & M_{i j}
\end{array}\right) .
$$

Upon diagonalization this mass matrix gives the usual seesaw spectrum of three light neutrinos $(\nu)$ with masses $m_{\nu} \sim m_{\mathrm{D}}^{2} / M$ and three heavy neutrinos $(N)$ with masses $\mathrm{O}(M)$.

It was pointed out long ago [18] that the mass matrix and the majoron coupling matrix for the light neutrinos will commute up to $\mathrm{O}\left(\left(m_{\mathrm{D}} / M\right)^{4}\right)$. Therefore, unless we make the heavy scale $v<\mathrm{O}(100 \mathrm{GeV})$ the lifetime of any neutrino $\nu$ with mass greater than $30 \mathrm{eV}$ will be too long to avoid the constraint from the age of the Universe, not to mention the more severe constraint on the lifetime from structure formation [19]. As a result, it has been generally accepted that additional Higgs fields must be added in order to get viable models for neutrino decay [20]. However, it has been argued [21] that the result in ref. [18] does not hold in general. Radiative corrections may generate finite pieces of $\mathrm{O}\left(m_{\mathrm{D}}^{2} /\left(8 \pi^{2} M^{2}\right)\right)$ to the off-diagonal part of the light neutrino couplings which will not be the same as 


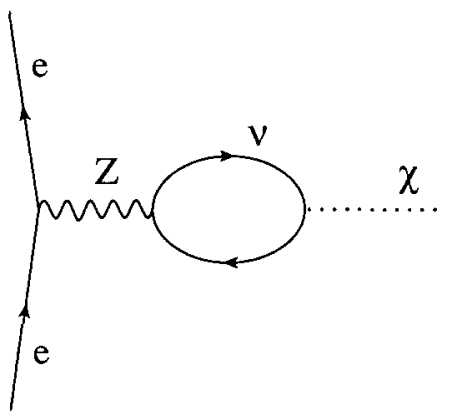

Fig. 1. Leading contribution to $g_{\mathrm{ee} x}$.

the corrections of the off-diagonal terms of the mass matrix *. The light neutrino lagrangian may then be written with derivative couplings as

$$
L_{\nu}=\frac{1}{2} m_{\nu, i} \bar{\nu}_{i} \nu_{i}+\frac{\partial_{\mu} \chi}{4 v}\left(\bar{\nu}_{i} \gamma^{\mu} \gamma_{5} \nu_{i}+\sum_{i j} \bar{\nu}_{i} \gamma^{\mu}\left(g_{\mathrm{v}, i j}+g_{\mathrm{a}, i j} \gamma_{5}\right) \nu_{j}\right),
$$

where $g_{v}$ and $g_{a}$ could be of order $\mathrm{O}\left(m^{2} /\left(8 \pi^{2} M^{2}\right)\right)$ and there may be vector off-diagonal couplings, but $g_{\mathrm{v}, i i}=0$. The decay rate for the heaviest of the light neutrinos into a particular channel is then given by

$$
\Gamma=\frac{m_{\nu}}{64 \pi}\left(\frac{m_{\nu}}{v}\right)^{2}\left(g_{\mathrm{v}, i j}^{2}+g_{\mathrm{a}, i j}^{2}\right),
$$

where we have neglected the mass of the final state neutrino.

In the singlet majoron model, coupling to other fermions arises through an intermediate $\mathrm{Z}$ boson and a neutrino loop, see fig. 1. The electron coupling in pseudoscalar form is then

$$
g_{\mathrm{ee} \chi} \approx \frac{G_{\mathrm{F}} m_{\mathrm{e}} m_{\nu}}{16 \pi^{2}}
$$

The most important constraint on this coupling arises from consideration of the cooling of red giant stars [8], $g_{\operatorname{ce}_{\chi}}>10^{-13}$.

\section{The singlet majoron model with explicit symmetry breaking}

Assuming that short distance physics only respects local gauge symmetries, the only way to have Nambu-Goldstone bosons appear in the spectrum is to ensure

* It is also possible to get rapid decay if one chooses the global symmetry to be a linear combination of the lepton numbers $\left(L_{i, \mu, \tau}\right)$ as opposed to just $L$ [22]. Rapid decay into three neutrinos is possible in models with Dirac neutrino masses and extra singlet scalars which do not acquire VEVs [23]. 
that there is an accidental (clearly a misnomer in this case) symmetry. There is no way to prevent operators which break the accidental symmetry from appearing at some level, but we can arrange for these operators to have dimension greater than four. In this case the symmetry breaking operators will be suppressed by powers of $M_{\mathrm{pl}}$ and a small mass is induced for the Nambu-Goldstone boson.

To accomplish this for majoron models, we gauge $\mathrm{U}(1)_{\mathrm{B}-\mathrm{L}}$ and include in the spectrum two singlet scalar fields. The first, $\phi_{1}$, plays a similar role to $\phi$ in the singlet model of sect. 2. This field has B $-\mathrm{L}$ quantum number $q_{1}=2$, allowing it to have a Yukawa coupling with the singlet neutrinos. The $\mathrm{U}(1)_{\mathrm{B}-\mathrm{L}}$ charge of the additional scalar field, $\phi_{2}$, is chosen such that the lowest dimensional B-L invariant operator which breaks the accidental U(1) has dimension greater than four. To leave matters as general as possible we allow $\phi_{2}$ to have fractional B $-\mathrm{L}$ charge. As such, there will be several different models possible for a given dimension; for example, $q_{2}=\frac{1}{2}, \frac{4}{3}, 3$, or 8 , will result in a dimension-five operator suppressed by one power of $M_{\mathrm{p}}$. As examples of $q_{2}$ assignments that do not work, $q_{2}=1$ or 4 allow a Planck scale cubic term in the lagrangian and the global symmetry would disappear entirely from the low energy lagrangian.

We write the lowest dimensional symmetry breaking term as

$$
L_{\text {S.B. }}=\frac{g_{\text {grav }} \phi_{1}^{n_{1}} \phi_{2}^{* n_{2}}}{M_{\mathrm{pl}}^{d}}+\text { h.c. }
$$

where $g_{\text {grav }}$ is the dimensionless strength of the operator, $d=n_{1}+n_{2}$ is the dimension of the symmetry breaking operator, and $n_{1}$ and $n_{2}$ are integers. Gauge invariance imposes the constraint

$$
n_{1} q_{1}-n_{2} q_{2}=0
$$

and to ensure that $d>4$ we require

$$
a q_{1}-b q_{2} \neq 0: \text { if } a+b \leqslant 4 ; \quad a, b \in \text { integers. }
$$

By choosing an appropriate Higgs potential both singlets will receive vacuum expectation values, $v_{1}$ and $v_{2}$ respectively. Given that $\mathrm{B}-\mathrm{L}$ is a local gauge symmetry there will be a lower bound on the $Z^{\prime}$ mass of $O(300 \mathrm{GeV})$ which, for reasonable $U(1)_{B-L}$ gauge couplings, will also be a lower bound on the larger of the two singlet VEVs. Had we chosen to gauge an anomaly free combination of lepton numbers this constraint would be relaxed [24].

Upon symmetry breaking, one combination of the phases of the singlets becomes the longitudinal part of the $Z^{\prime}$ gauge boson, while the orthogonal combination becomes the majoron,

$$
\chi=\frac{q_{2} v_{2} \chi_{1}-q_{1} v_{1} \chi_{2}}{\left(q_{2}^{2} v_{2}^{2}+q_{1}^{2} v_{1}^{2}\right)^{1 / 2}} .
$$


The diagonal majoron couplings to the light neutrinos are given in the pseudovector form as

$$
L_{\nu \nu \chi}=\frac{q_{2} v_{\mathrm{eff}}}{v_{1}} \frac{\partial_{\mu} \chi}{4 v_{1}}\left(\bar{\nu}_{i} \gamma^{\mu} \gamma_{5} \nu_{i}\right)
$$

where the first factor accounts for the mixing of $\phi_{1}$ and $\phi_{2}$ in eq. (14), and for convenience, we define

$$
v_{\mathrm{eff}}^{2}=\frac{v_{1}^{2} v_{2}^{2}}{q_{1}^{2} v_{1}^{2}+q_{2}^{2} v_{2}^{2}} .
$$

The explicit symmetry breaking will induce a mass for the majoron,

$$
m_{\chi}^{2}=2 g_{\text {grav }} \frac{v_{1}^{n_{1}} v_{2}^{n_{2}}}{M_{\mathrm{pl}}^{d-4}} \frac{n_{2} n_{1}}{q_{2} q_{1} v_{\mathrm{eff}}^{2}} .
$$

The couplings of other fermions to the majoron arise from the same diagram as in the simple model of sect. 2. At first it might appear that one could replace the $\mathrm{Z}$ boson by a $Z^{*}$ boson, while at the same time replacing the light neutrino loop by a loop with a heavy singlet neutrino. If such diagrams existed they would dominate over the $\mathrm{Z}$ exchange by a ratio of heavy to light neutrino masses, however, one can show that such contributions vanish. First, the $\chi-Z^{\prime}$ mixing (through a heavy singlet neutrino loop) cannot lead to an $\bar{f} \gamma_{5} f \chi$ vertex since $\mathrm{B}-\mathrm{L}$ is a vectorial symmetry. There will be $Z-Z^{\prime}$ mixing at one-loop via the exchange of charged fermions and, since $Z$ coupling to matter is parity violating, $\bar{f} \gamma_{5} f \chi$ may be induced as a two-loop effect. However, such diagrams also give vanishing contribution. This is best seen by working in the Landau gauge, where the gauge boson propagator is purely transverse. There is no $\chi-Z^{\prime}$ or $\chi-Z$ mixing in this gauge, so only the unphysical Higgs may contribute; but this scalar is necessarily an $\mathrm{SU}(2)_{\mathrm{L}}$ singlet (since it has to couple to the heavy singlet neutrino) and so has no coupling to the standard model fermions.

The constraint from coupling majorons to ordinary matter is therefore the same as in the simplest model, with the exception that even this mild constraint does not hold if $m_{\xi} \geq 10 \mathrm{keV}$, since the majorons will be too heavy to be produced in red giant cores.

\section{Majoron contributions to the energy density}

In theories where a global symmetry is broken spontaneously as well as explicitly by a term with a relatively small coupling, there are two contributions to 
the energy density in Goldstone bosons, the thermal production due to interactions with the other particles in the medium and a coherent oscillation of the Goldstone field if the initial angle of that field does not align with the direction of explicit symmetry breaking. The coherent contribution may be enhanced by the presence of strings and walls associated with spontaneous symmetry breaking, or may be suppressed by an inflationary epoch. Both terms may be alleviated if the Goldstone bosons decay rapidly.

We begin by summarizing the cosmological history of the model. For this task it is convenient to refer to the scalar fields by the magnitude of their VEVs, in which case we will refer to the smaller VEV (and its field) by the subscript $a$ and the larger VEV by the subscript $b$. It may be that $a \equiv 1$, indicating the scalar field that couples to neutrinos, or that $a \equiv 2$, depending upon the scenario. Then, the larger of the VEVs, $v_{b}$, is established first, the associated scalar is "eaten" by the $\mathrm{U}(1)_{\mathrm{B}-\mathrm{L}}$ gauge field, and the $\mathrm{Z}^{\prime}$ gets a mass. At this time a set of local U(1) gauge strings will form.

When the remaining global U(1) symmetry is broken, $v_{a}$ is established and the majoron becomes a light degree of freedom, breaking the global symmetry results in a set of global cosmic strings. Additionally, the local gauge strings present from the breaking of $\mathrm{U}(1)_{\mathrm{B}-\mathrm{L}}$ may become catalysis sites for another set of global strings.

The majoron has a small mass (eq. (17)) which is present immediately at the breaking of $\phi_{a}$. This mass becomes dynamically important at a time labeled by the temperature $T_{\mathrm{m}}$, defined by

$$
m_{\chi}=3 H\left(T_{\mathrm{m}}\right),
$$

where $H$ is the expansion rate of the Universe. It is possible that $m_{\chi}>H$ at the time when $v_{\mathrm{a}}$ is established, in which case $T_{\mathrm{m}} \approx v_{\mathrm{a}}$. In either case, two things occur at $T_{\mathrm{m}}$. First, one may expect a contribution to the energy density from a non-thermal population of long-wavelength coherent majorons. Second, the global cosmic strings become the edges of dynamically important domain walls, whose thickness is of order $1 / m_{\chi}$. Depending upon details of the model these string-wall networks may or may not go away.

At first the majoron field should be thermally coupled to the plasma through scattering processes, but at some time (labeled by the temperature $T_{\mathrm{d}}$ ) the majorons will decouple. It is possible that decays and inverse decays may recouple the majorons to the plasma at a later date. The rate of inverse decays in a relativistic plasma is $\sim \Gamma_{\chi} m_{\chi} / T$, whereas the expansion rate is $T^{2} / M_{\mathrm{pl}}$. Evidentially, inverse decays can bring a species back into equilibrium at low temperatures - depending on the interaction strength. If the majoron-neutrino coupling is weak then full equilibrium will not be achieved until the majorons are non-relativistic at which time their energy density will be dumped into neutrinos but back-reactions will be unimportant. Of course, this may occur after the present epoch. 


\subsection{THERMAL CONTRIBUTION TO THE ENERGY DENSITY}

If the majorons are lighter than a few $\mathrm{eV}$ their thermal contribution to the energy density will not be significant, but a larger mass is certainly possible in the present context. The energy density in a thermal relic population of majorons is

$$
\rho_{\mathrm{th}}=n_{\mathrm{th}} m_{\chi}=\frac{\zeta(3)}{2 \pi^{2}} T_{\chi}^{3} m_{\chi},
$$

where $T_{\chi}$ is the "temperature" of the majorons. Generally, $T_{\chi}$ will differ from the photon temperature by a factor $R_{\chi}$, in which case the thermal relic density can be given in terms of the critical density for closure, $\rho_{\text {crit }}$, as

$$
\Omega_{\chi, \mathrm{th}} \equiv \frac{\rho_{\mathrm{th}}}{\rho_{\text {crit }}}=19.8 \frac{m_{\chi}}{\mathrm{keV}} \frac{R_{\chi}^{3}}{h_{0}^{2}},
$$

where $h_{0}$ is the expansion rate in units of $100 \mathrm{~km} / \mathrm{s} \mathrm{Mpc}$, and we used a microwave background temperature of $2.74 \mathrm{~K}$. If the Universe has expanded adiabatically since the majorons decoupled, and light neutrinos are the only non-photonic species present today then

$$
R_{\chi}^{3}=\left(\frac{T_{\chi}}{T_{\gamma}}\right)^{3}=\frac{g_{\gamma}}{g_{\gamma}+g_{\mathrm{e}}} \frac{g_{\gamma}+g_{\mathrm{e}}+g_{v}}{g_{*}} \approx 5.8 \times 10^{-2},
$$

where $g_{\gamma}=2, g_{\mathrm{e}}=\frac{7}{2}, g_{\nu}=\frac{21}{4}$, and $g_{*}$ is the total number of relativistic degrees of freedom other than majorons at the time that the majorons decouple.

As will be shown below, for most scenarios decoupling may be expected to occur soon after $\phi_{a}$ develops a VEV. At first the self-couplings of the majorons and $\rho$ particles and their coupling to singlet neutrinos may be expected to keep the majorons in equilibrium. If $v_{a}$ and $v_{b}$ are not too dissimilar, the $\mathrm{B}-\mathrm{L}$ gauge interactions will couple the singlet neutrinos, majorons and $\rho$ particles to the ordinary degrees of freedom in the plasma. However, all these particles, except for the majoron, presumably have masses of order $v_{a}$ or $v_{b}$, and will soon freeze out of the primordial plasma. After they freeze out the only way to avoid decoupling is through the light neutrinos. The most direct reaction to maintain equilibrium is $\nu \nu \leftrightarrow \chi \chi$ through t-channel exchange of a neutrino. The cross section for this process is given by

$$
\sigma_{\nu \nu \rightarrow \chi \chi}=\frac{1}{64 \pi} \frac{m_{\nu}^{2}}{v_{1}^{4}}\left(\frac{q_{2} v_{\mathrm{eff}}}{v_{1}}\right)^{4} .
$$

This result applies in the limit where the $\rho$ particles are much heavier than the momentum exchange in the reaction. The last factor arises from projecting the 
majoron onto the scalar field that couples to the neutrinos. Allowing this factor to be of order unity, the decoupling point is given by

$$
\frac{T_{\mathrm{d}}}{v_{1}} \sim 10^{2}\left(\frac{1 \mathrm{eV}}{m_{\nu}}\right)^{2}\left(\frac{v_{1}}{1 \mathrm{GeV}}\right)^{3}
$$

Unless $l_{1}$ is quite small and the neutrino masses are large the light neutrinos cannot keep the majorons in equilibrium after the phase transition. The converse of this is that the constraint on majoron models from big-bang nucleosynthesis is fairly weak (see sect. 6). Thus, except for exceptional circumstances, it is reasonable to assume that the majorons decouple soon after the phase transition.

Eq. (20) is valid only so long as the majorons are stable. However, if majorons have a mass then they may decay with a rate

$$
\Gamma_{\chi}=\frac{m_{\chi}}{16 \pi} \sum_{i} g_{2 v_{i}},
$$

where

$$
g_{\nu_{i}}=\frac{m_{\nu_{i}}}{l_{1}} \frac{q_{2} l_{\mathrm{eff}}}{v_{1}}
$$

is the diagonal coupling to neutrino $i$ in its pseudoscalar form. The sum is over neutrino species lighter than the majoron. Off-diagonal couplings are presumably less important. The decay rate depends on the light neutrino masses, which arise through the seesaw mechanism. Phenomenological constraints on neutrino masses will impose constraints on the majoron decay rate.

Some general remarks: (a) If neutrino masses are small enough, then majorons are stable for all practical purposes. (b) If the majorons decay well after they become non-relativistic, the decay neutrinos will contribute to the energy density in radiation, and this can produce a conflict between theories of galaxy formation and limits on the fluctuations in the temperature of the cosmic background radiation. In the extreme case the density of decay products can overclose the Universe. (c) If the majorons decay too quickly then inverse decays may recouple the majoron field to the plasma before nucleosynthesis begins at a time of about $1 \mathrm{~s}$, or a temperature $T_{\mathrm{BBN}} \approx 1 \mathrm{MeV}$. In this case an excess of ${ }^{4} \mathrm{He}$ will be produced (see sect. 6). (d) If majorons are more massive than about $10 \mathrm{MeV}$ then their decay before nucleosynthesis (more specifically - before the neutrinos decouple) poses no significant cosmological problems other than a potential increase in entropy and consequent dilution of baryon number.

Let us investigate the possibility that relic majorons form warm dark matter. As an example, consider the case where the two VEVs are comparable, $v_{1}=v_{2}=v$. We vary $q_{2}$, the $\mathrm{B}-\mathrm{L}$ charge of $\phi_{2}$, so as to get the appropriate dimension 
operator. To get the majoron to be warm dark matter we want the mass of the majoron to be of order $1 \mathrm{keV}$. Using eq. (17) and assuming a dimensionless strength of order unity for the explicit symmetry breaking, we find a relation between $v$ and the dimension of the operator,

$$
\log _{10}\left(\frac{v}{1 \mathrm{GeV}}\right) \sim \frac{19(d-4)-12}{d-2}
$$

The decay rate will be given by eq. (24). If we scale to a neutrino mass of $10 \mathrm{eV}$, keep the majoron mass at $1 \mathrm{keV}$, and drop the numerical factor $\left(q_{2} v_{\text {eff }} / v_{1}\right)^{2}$ then the majoron lifetime is

$$
\tau_{\chi} \sim 3 \times 10^{5}\left(\frac{v}{\mathrm{TeV}}\right)^{2}\left(\frac{10 \mathrm{eV}}{m_{\nu}}\right)^{2} \frac{\mathrm{keV}}{m_{\chi}} \mathrm{s}
$$

If $d=5$ then $v \sim 200 \mathrm{GeV}$ and the lifetime is around $10^{4} \mathrm{~s}$. Majorons have mass but decay quickly so they do not constitute dark matter. For keV majorons, their decay rate lies in an acceptable window which poses no problems for galaxy formation or for nucleosynthesis. If $d=6$ then $v \sim 3 \times 10^{6} \mathrm{GeV}$ and the lifetime is around $3 \times 10^{12} \mathrm{~s}$. This decay rate is troublesome for galaxy formation, but a modest decrease in the heaviest neutrino mass would allow the majoron to be sufficiently stable to be dark matter. For $d=7$ we find $v=10^{9} \mathrm{GeV}$ and lifetimes longer than the age of the Universe, so majorons can play the role of warm dark matter in such models. Higher $d$ also admit a value of $\Omega_{\chi} \sim 1$ in stable thermal relics. Clearly, we could vary the ratio of the two VEVs, the dimensionless strength of the gravitational operator, etc., providing even more flexibility to the models.

Let us look at the $d=5$ case a little more closely. This can be achieved by $q_{1}=2$ and $q_{2}=\frac{1}{2}, \frac{4}{3}, 3$, or 8 . Let us concentrate on $q_{2}=\frac{1}{2}$, and still take the two VEVs to be the same. Assuming that both VEVs develop before the standard model breaks from $\mathrm{SU}(2) \times \mathrm{U}(1)_{\mathrm{Y}}$ down to $\mathrm{U}(1)_{\mathrm{em}}$, so that $g_{*}=67 \frac{3}{8}$, we find $R_{\chi}=0.058$. For a Hubble parameter of $h=0.5$ (where $h$ is $H$ in units of $100 \mathrm{~km} / \mathrm{s}$ $\mathrm{Mpc})$ and a majoron mass of $m_{\chi}=53 g_{\text {grav }}^{1 / 2} \mathrm{keV}(v / 1 \mathrm{TeV})^{3 / 2}$ this gives $\Omega_{\chi}=$ $242 g_{\text {grav }}^{1 / 2}(v / 1 \mathrm{TeV})^{3 / 2}$, if the majorons were stable. However, with these parameters and a $30 \mathrm{eV}$ neutrino mass, the lifetime of the majorons is of order $10^{4} \mathrm{~s}$.

Coincidentally, the temperature of the Universe at $10^{4} \mathrm{~s}$ is of order $10 \mathrm{keV}$, so the majorons would be mildly non-relativistic when they decay. The decay neutrinos would be slightly more energetic than the blackbody neutrinos, but not so much as to pose a problem for galaxy formation. However, as Akhmedov et al. [17] point out, if one assumes an upper limit on the neutrino mass of roughly $30 \mathrm{eV}$, then this argument yields an upper limit to the VEV, $v \leqq 10 \mathrm{TeV}$. Both $m_{x}$ and $\tau_{x}$ increase with $v$ and so the present density in relativistic decay neutrinos also increases; which eventually creates a problem for galaxy formation. In that analysis 
there is only one VEV, but in our models there is more flexibility. By separating $v_{1}$ and $v_{2}$ and choosing different $\mathrm{B}-\mathrm{L}$ charges one can maintain a short majoron lifetime while increasing $m_{\chi}$. For example, in the toy case we chose $q_{1}=2$ and $q_{2}=\frac{1}{2}$ so that varying $v_{1}$ and $v_{2}$ independently (but keeping $v_{1}<v_{2}$, so that $\left.v_{\text {eff }} \approx v_{1}\right)$ gives

$$
\begin{aligned}
& m_{\chi} \approx \frac{v_{1}^{-1 / 2} v_{2}^{2}}{M_{\mathrm{p} 1}^{1 / 2}}, \\
& \tau_{\chi} \approx \frac{8 \pi}{m_{\chi}}\left(\frac{v_{1}}{m_{v^{\prime}}}\right)^{2} .
\end{aligned}
$$

As $v_{2}$ is raised the majoron mass increases but the lifetime shortens since the neutrino couplings haven't been altered. The energy in relativistic decay products increases more slowly. There is still a prohibited region from BBN considerations, $0.1 \mathrm{MeV} \leq m_{\chi} \leq 10 \mathrm{MeV}$, but generally mıre massive majorons are still viable in a model with two VEVs.

Another interesting case occurs when the heaviest neutrino has a mass chosen to solve the solar neutrino problem via the MSW mechanism [25], $m_{v} \sim 10^{-2.5} \mathrm{eV}$. the majoron lifetime would be around $10^{12} \mathrm{~s}$ in such a model. This value is somewhat uncomfortable - the decay products would be relativistic and the Universe would be dominated by radiation for a period late in its history, making a consistent theory of galaxy formation more difficult. To rectify this, it would be desirable to make the majoron lighter and longer lived than the age of the Universe. This could be accomplished by increasing $v_{1}$ while holding $v_{2}$ fixed and the neutrino masses constant. This would decrease the majoron mass and their coupling to neutrinos.

Finally, one of the main motivations for majoron models is to allow for neutrino masses in excess of $30 \mathrm{eV}$. The presence of the massless majoron allows the heavy neutrino to decay into a lighter neutrino and a majoron with a short lifetime. The issue of off-diagonal couplings may be addressed by invoking radiative loops, or enlarging the model to include extra scalar fields so that the mass and coupling matrices are not nearly proportional. With the consideration of gravitational effects one may expect the majoron to get a mass, perhaps in the keV region. Neutrinos heavier than the majoron may still decay into a majoron and a lighter neutrino, but neutrinos less massive than the majoron, and yet more massive than $30 \mathrm{eV}$ have no obvious decay channel and would present a cosmological problem.

\subsection{COHERENT OSCILLATIONS}

The small explicit breaking of a spontaneously broken global symmetry leads to a coherent oscillation of the Goldstone field around its minimum. This phe- 
nomenon is well known in axion models [26] and leads to a constraint on the axion decay parameter, $f_{\mathrm{a}}$. If gravitational effects explicitly break the Peccei-Quinn symmetry in an axion model, then the energy in coherent oscillations is smaller than that in thermal axions unless the explicit symmetry breaking operators has $d \geqslant 10[12,13]$. The models considered here are similar except for the present two VEVs, but we will show that the second VEV can only make the coherent oscillations less important.

The majoron field is static until the mass becomes greater than the expansion rate of the Universe, $m_{\chi}=3 H\left(T_{\mathrm{m}}\right)$. The energy density in majorons at that time is

$$
\rho_{\mathrm{mis}}=\frac{1}{2} m_{\chi}^{2} \chi_{0}^{2} \frac{q_{2} q_{1} v_{\mathrm{eff}}^{2}}{n_{2} \pi_{1}}
$$

where $\chi_{0}$ is the initial misalignment angle, presumably of order unity. These coherent oscillations have a wavelength which is greater than their Compton wavelength and the energy density scales as non-relativistic matter. Taking into account the expansion of the Universe from $T_{\mathrm{m}}$ until today yields

$$
\Omega_{\chi} \approx 7 \times 10^{-20} \frac{g_{\mathrm{m}}^{3 / 4}}{h^{2}} \frac{m_{\chi}^{1 / 2} v_{\mathrm{eff}}^{2}}{\mathrm{GeV}^{5 / 2}} \frac{q_{2} q_{1}}{n_{2} n_{1}},
$$

where $g_{\mathrm{m}}$ is the number of equilibrated degrees of freedom at $T_{\mathrm{m}}$.

To compare the thermal relic and coherent energy densities we compare their contributions to the majoron number density at the temperature $T_{\mathrm{m}}$. Dropping all dimensionless factors, the ratio is given by

$$
R_{n_{\chi}} \equiv \frac{n_{\mathrm{mis}}}{n_{\mathrm{th}}} \approx \frac{m_{\chi} v_{\mathrm{eff}}^{2}}{m_{\chi}^{3 / 2} M_{\mathrm{pl}}^{3 / 2}} \approx\left(\frac{v_{a}}{M_{\mathrm{pl}}}\right)^{(10-d) / 4}\left(\frac{v_{a}}{v_{b}}\right)^{n_{b} / 4}
$$

where we have used $T_{\mathrm{m}} \approx\left(m_{\chi} M_{\mathrm{pl}}\right)^{1 / 2}$ in the first relation; and eq. (17) for the majoron mass and eq. (16) for $v_{\text {eff }}$ in the second. Note that the result depends only on the relative magnitude of the VEVs and not on which one couples to neutrinos. The power $(10-d) / 4$ is the same as in the axion result. The extra ratio of VEVs reflects the fact that a large $v_{b}$ will increase $m_{\chi}$ without affecting $v_{\text {eff }}$, but this can only reduce $R_{n_{x}}$. Finally, for $d=5$ (and maybe for $d=6$ ) $T_{\mathrm{m}} \approx v_{\text {eff }}$, i.e. the majoron mass is greater than the expansion rate at the moment of the phase transition. In this case $R_{n_{x}} \approx m_{x} / v_{\text {eff }}$, and, again, misalignment is unimportant. Thus, the misalignment energy is only important if the dimension of the gravitational operator is $d \geqslant 10$.

Eq. (31) may need to be modified to account for the majorons that arise from cosmic strings. Following the arguments made by Davis and Shellard [27] for axion models, the presence of global strings may be expected to increase the number of 
majorons in the coherent field by perhaps an order of magnitude due, primarily, to the logarithmic divergence in the string tension of global strings. However, this conclusion depends on the spectrum of Goldstone bosons radiated from cosmic string loops at the time when $T=T_{\mathrm{m}}$ [28]. A conclusion to the argument awaits a definitive numerical analysis of the radiation spectrum.

The derivation of eq. (31) also assumed that the initial misalignment angle was of order one. If there were an inflationary epoch between the breaking of the global U(1) symmetry and $T_{\mathrm{m}}$ then the majoron field could be correlated over horizon size regions and could, by chance, be near its minimum. This would yield a reduction in the misalignment energy density from the estimate in eq. (30) due to a suppression of $\chi_{0}$. The reduction is limited by quantum fluctuations in the majoron field during the inflationary epoch to a factor of order $\left(H_{\mathrm{I}} / v_{\text {eff }}\right)^{2}$, where $H_{\mathrm{I}}$ is the expansion rate during inflation. One must also check that isocurvature density perturbations induced by these fluctuations are not in conflict with observations of the cosmic microwave background radiation.

One may wonder if the presence of a small explicit symmetry breaking can cause the initial angle of the majoron field to lie near the minimum of the majoron potential, i.e. $\chi_{0} \approx 0$. The same issue is relevant for the question of the formation of majoron strings and domain walls (see below). We believe the answer is no, the angular minimum is not strongly preferred. The essential point is that the radial gradient of the potential will be much greater than the angular gradient caused by the explicit symmetry breaking, see fig. 2. Once a VEV has been established, it will roll radially outward much faster than it can slip sideways. The one possibility seems to be that the tilt causes the VEV to be established in a direction aligned with the explicit symmetry breaking, so that the radial roll of $\phi$ takes one directly to the global minimum. To study this, we consider a first order phase transition which proceeds through the formation of bubbles, which may form by quantum tunneling or by thermal fluctuations. We discuss the tunneling case first and comment on thermal fluctuations later.

The rate of bubble formation depends upon the action to form a bubble of zero energy, which in turn depends upon the amount of supercooling and the shape of

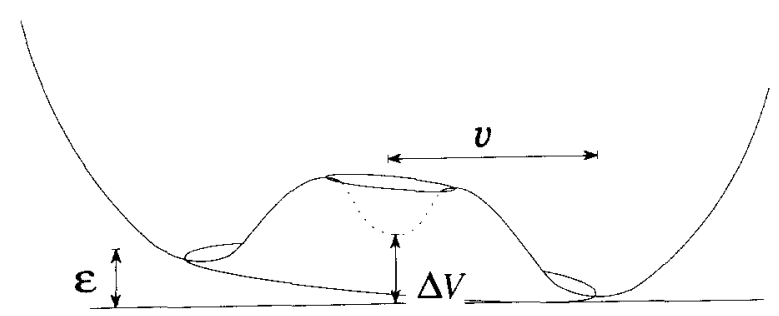

Fig. 2. Scalar potential showing small explicit breaking. 
the potential. Dimensional analysis suggests that the action for a critical size bubble is

$$
A_{\mathrm{c}} \sim R_{\mathrm{c}}^{4} \Delta V \sim R_{\mathrm{c}}^{3} \sigma \sim \frac{\sigma^{4}}{(\Delta V)^{3}},
$$

where $R_{\mathrm{c}} \sim \sigma / \Delta V$ is the radius of a critical size bubble, $\Delta V$ is the potential difference between the false and truc vacua, $\sigma \sim v^{2} / l \sim \Delta V \sim v(\Delta V)^{1 / 2}$ is the surface tension on the bubble, $l \sim v /(\Delta V)^{1 / 2}$ is the surface thickness, and $v$ is the magnitude of the VEV to be established after the phase transition. The formation rate per unit volume scales as

$$
\Gamma_{\mathrm{c}} \sim R_{\mathrm{c}}^{-4} \exp \left(-A_{\mathrm{c}}\right) .
$$

This rate should be compared to the expansion rate of a horizon volume, $\sim H^{4}$. Now, even though the prefactors may have ratios of large numbers such as $M_{\mathrm{pl}} / T$, this comparison will be dominated by the exponential. When supercooling first begins $\Delta V$ will be quite small, the action for bubble formation will be large, and bubble formation will be slow compared to the expansion rate. However, as the Universe continues to expand and supercool, $\Delta V$ will increase and $A_{\mathrm{c}}$ will decrease. Eventually, $A_{c}$ will be of order a few hundred and bubble formation will become rapid. The significant point for us is that by the time this happens the tilt of the potential will be rather small, $\epsilon \ll \Delta V$. As a result the action to form a critical bubble with the majoron field at a local maximum will be of order $R_{\mathrm{c}}^{4}(\epsilon+\Delta V)$ whereas the action to form a bubble at the global minimum of the potential energy is just $R_{\mathrm{c}}^{4} \Delta V$. Since bubble formation proceeds when $A_{\mathrm{c}} \sim$ a few hundred, we require that $\epsilon / \Delta V \sim 10^{-3}$ in order to significantly bias bubble formation. Since $\Delta V$ is of order $\lambda v^{4}$, this requires a very small self-coupling for the scalar fields, which in the context of a renormalizable U(1) local gauge theory is not natural. We conclude that for the case of quantum tunneling the majoron field is randomly aligned with respect to the explicit symmetry breaking.

The thermal fluctuation case is similar. Instead of considering $\exp \left(-A_{\mathrm{c}}\right)$, one considers $\exp \left(-\beta F_{\mathrm{c}}\right)$, where $F_{\mathrm{c}}$ is the free energy of a thermal fluctuation forming a bubble for which the energy is maximal, $\mathrm{d} E / \mathrm{d} R=0$. Which process dominates depends on the ratio $\beta F_{\mathrm{c}} / A_{\mathrm{c}}$, which in turn depends on the details of the theory. Generally, the transition for a weakly self-coupled scalar field should be dominated by thermal fluctuations, whereas for a strongly coupled field the tunneling process should win out. In either case, the explicit breaking due to gravity should not be important. Therefore, for a first order phase transition, even though the majoron mass may be greater than the expansion rate at the time of symmetry breaking, there will not be any suppression of the coherent energy density due to a physical alignment of the majoron field along a preferred direction, and eq. (31) may be used. 
As with the thermal majorons there is the possibility of majoron decay; however, if we confine ourselves to the case where the coherent energy is more important than the thermal energy we can show that majoron decay is not a relevant issue. By using the ratio of coherent to thermal majoron densities, $R_{n_{x}}$, given in eq (32), we can express the majoron mass as

$$
m_{\chi}=\frac{10^{-8} \Omega_{\chi}}{R_{n_{\chi}}} \mathrm{GeV} .
$$

Using this form in eq. (31) gives

$$
v_{\mathrm{eff}}=10^{8.5} \Omega_{\chi}^{1 / 2} R_{n_{x}}^{1 / 4} \mathrm{GeV}
$$

To maximize the decay rate we take the neutrino mass to be comparable to the majoron mass, take $v_{1}=v_{\text {eff }}$, and find

$$
\tau_{\chi} \geq \frac{10^{17} R_{n_{\chi}}^{7 / 2}}{\Omega_{\chi}^{5 / 2}} \mathrm{~s} .
$$

Decreasing the lifetime requires either a large value of $\Omega_{\chi}$ with a corresponding unacceptable density of decay neutrinos, or requires making a thermal density of majorons in excess of the coherent density.

\section{Majoron strings and walls}

In the models presented here there are several types of topological defects which may occur. When $\mathrm{U}(1)_{\mathrm{B}-\mathrm{L}}$ breaks, a set of local cosmic strings forms with string tension $\mu_{b} \sim v_{b}^{2}$. We call these type- $b$ strings since they have a defect in the field $\phi_{b}$. Later, when $T \sim v_{a}$, the second U(1) breaks and type- $a$ global strings form with $\mu_{a} \sim v_{a}^{2}$. At the same time, type- $b$ strings develop a defect in $v_{a}$ with winding number adjusted to minimize the energy in the angular gradients of the fields. Since there is explicit symmetry breaking in our models, at the time $T_{\mathrm{m}}$ one expects domain walls to form with surface tension $\sigma \sim m_{x} l_{\text {eff }}^{2}$. These domain walls may exist as isolated surfaces or be bounded by strings. If $m_{\chi}>H\left(v_{a}\right)$ these walls will exist from the moment that majorons exist, but otherwise they form at a later time.

The fate of the string-wall network depends upon how many walls are connected to the various types of strings. If either the type- $a$ or the type- $b$ strings come with a single wall attached, then the system can dissipate by self-intersections [29]. If, however, both types of strings have a number of walls $N_{\text {wall }}>1$, then the strings cannot annihilate and the walls may eventually dominate the energy density 
of the Universe. The energy density in a single wall stretching across todays' horizon is

$$
\Omega_{\text {wall }} \sim \frac{\sigma H}{H^{2} M_{\mathrm{pl}}^{2}} \sim 10^{4} \frac{m_{\chi} v_{\mathrm{eff}}^{2}}{\mathrm{GeV}^{3}}
$$

A single wall stretching across the horizon would be likely to contribute to the quadrupole moment of the microwave background, and so it seems reasonable to require $\Omega_{\text {wall }} \lesssim 10^{-5}$. The resulting constraint, $m_{\chi} v_{\text {eff }}^{2} \leqq 10^{-9} \mathrm{GeV}^{3}$ is much stronger than that which follows from the coherent energy density (eq. (31)), and somewhat stronger than the constraint from thermal relics, depending on the magnitude of $v_{\text {eff }}$. For example, it is violated by $\sim$ seven orders of magnitude for the toy model used in the presentation of the thermal relics. If majorons are to make a significant contribution to $\Omega$, the model must be defined so that $N_{\text {wall }}=1$ for some set of strings. On the other hand, it is not difficult to suppress $m_{\chi}$ by sufficient powers of $M_{\mathrm{pl}}$ in order that majoron walls pose no problem.

Constructing models where $N_{\text {wall }}=1$ for either type- $a$ or type- $b$ strings may be accomplished by an appropriate choice of the $\mathrm{B}-\mathrm{L}$ charges $q_{a}$ and $q_{b}$. The problem is identical to that considered in ref. [13] for a class of axion models, so we summarize that analysis. The energy density in the majoron field around a string is proportional to

$$
\left(\frac{\partial \chi}{\partial \theta}\right)^{2} \sim\left(q_{b} w_{a}+q_{a} w_{b}\right)^{2} \sim\left(n_{b} w_{b}+n_{a} w_{a}\right)^{2},
$$

where $w_{a}$ and $w_{b}$ are the winding numbers for $\phi_{a}$ and $\phi_{b}$ respectively. Recall that $n_{a} / n_{b}=q_{b} / q_{a}$, with both $n_{a}$ and $n_{b}$ chosen to be integers with no common factors other than one. At the same time, the number of walls is given by the number of maxima in the gravitationally induced potential (eq. (11)),

$$
N_{\mathrm{wall}}=n_{b} w_{b}+n_{a} w_{a} .
$$

Therefore, minimizing the field energy reduces the number of walls to a minimum as well.

For minimal type- $b$ strings we have $w_{b}=1$, and $w_{a}$ is chosen to minimize the gradient energy; while for type- $a$ strings $w_{b}=0$ and $w_{a}=1$. There are three possibilities for achieving strings with but one wall. (a) If $n_{b}=1$, type- $b$ strings have $w_{a}=0$ and one wall. (b) Similarly, if $n_{a}=1$, then type- $a$ strings have a single wall. (c) It is possible for $n_{a} \neq 1$ and $n_{b} \neq 1$ but to still have that that $w_{a}$ which minimizes eq. (39) results in a single wall; e.g. if $q_{a}=2$ and $q_{b}=\frac{6}{5}$, then $n_{b}=5$, $n_{a}=3$, and $w_{a}=2$.

Even if the network can dissipate, there is an additional constraint that this happens quickly enough. The system cannot dissipate until the energy in walls is 
comparable to the energy in strings. If the string energy dominates, then small loops of string that intersect walls result in holes which are subcritical in size; i.e. they do not expand to eat up the wall, but rather the hole shrinks and fills in. In order to get dissipation the critical size hole must be less than the horizon size. For type- $b$ strings, this occurs when $\left(v_{b} / c_{a}\right)^{2}\left(H / m_{\chi}\right)>1$. This may be later than the time when the majoron mass first becomes effective due to the difference in the magnitude of the VEVs that control the characteristics of the walls and the strings. If this time occurs after the time of matter-radiation decoupling, and $v_{b} \geq 10^{16}$ $\mathrm{GeV}$, then the strings still present at that time will cause excess fluctuations in the microwave background.

There are additional constraints that arise from the decay products of the strings and walls. For example, there are the majorons created in the dissipation of the type- $a$ strings, which strengthen the constraint from the misalignment energy, eq. (31). Other examples concern the type- $b$ strings. After they first form and before they get dressed up with a majoronic cocoon at $T \sim v_{\alpha}$, a scaling solution is established and presumably maintained by the radiation of gravitons. Requiring that the graviton energy not contribute too much density during nucleosynthesis provides a constraint on $v_{b}, v_{b} \ln \left(v_{b} / v_{a}\right) \lesssim 10^{18} \mathrm{GeV}$ [30]. Similarly, if $n_{a}=1$, type- $b$ strings develop no walls at all for $w_{a}=-n_{b}$. In this case the number density of type- $b$ strings is always determined by a scaling solution. Requiring that density fluctuations induced by these strings do not cause excess fluctuations in the microwave background implies $v_{b} \leq 10^{16} \mathrm{GeV}$.

\section{Thermal majorons and nucleosynthesis}

If majorons are in thermal equilibrium at $T=1 \mathrm{MeV}$ they will contribute to the energy density an amount equivalent to $\frac{4}{7}$ of a neutrino species. However, Walker et al. [31] argue that based on a determination of the primordial Helium abundance the excess energy density cannot exceed the standard cosmology by more than 0.3 species, i.e. $\Delta N^{\mathrm{BB}} N_{v}<0.3$. As pointed out earlier there are two ways for neutrinos to come into equilibrium - either through scattering events or through decays and inverse decays. Bertolini and Steigman [7] have examined the scattering case for the simple singlet model discussed in sect. 2 . Using the cross section of eq. (22) we adapt their result to the model in sect. 3 and find

$$
\frac{m_{\nu}}{\mathrm{MeV}}\left(\frac{\mathrm{GeV}}{v_{1}}\right)^{2}\left(\frac{q_{2} v_{\mathrm{eff}}}{v_{1}}\right)^{2}<4.6 \times 10^{-5},
$$

where the last factor accounts for the fact that the majoron does not necessarily couple to the neutrinos. If eq. (41) is satisfied the majorons freeze out before $T \sim 100 \mathrm{MeV}$ and, as a result, the entropy released when the pions and muons 
freeze out dilutes the majorons and their contribution to the density is less than 0.3 neutrino species. If the majorons freeze out later they share in the entropy release and the Helium abundance does not come out right.

The other constraint comes from decays and inverse decays [32]. As a safe constraint, we require that inverse decays come into equilibrium after the temperature drops below $5 \mathrm{MeV}$. If the majorons come into equilibrium before that, then we can be sure that they would share in the entropy of the Universe before nucleosynthesis begins and before the neutrinos themselves freeze out. If the inverse decays do not become effective until later they will only share the neutrino energy density. The effects on Helium production are more difficult to calculate (see, for example, Dodelson and Turner [33] for a discussion of the subtleties in phase space distributions during neutrino freeze out). We therefore take as an approximate condition

$$
\Gamma_{i} \frac{m_{i}}{3 \times 5 \mathrm{MeV}} \leq H(5 \mathrm{MeV}),
$$

where the subscript $i$ indicates either neutrino or majoron depending upon which is heavier, $\Gamma_{i}$ is the decay rate at rest, and the second factor accounts for the Lorentz dilation of the decay of relativistic particles. The factor of 3 multiplying the temperature crudely accounts for the average energy of a massless species. We ignore cases where two particles are nearly degenerate in mass, and we take no account of quantum statistics (Pauli blocking, Bose enhancement) in determining our criteria. Evaluating eq. (42) at $T_{\mathrm{d}}=5 \mathrm{MeV}$ when the number of degrees of freedom is $g_{\text {eff }}=\frac{43}{4}$,

$$
\frac{\mathrm{MeV}}{m_{i}} \frac{\mathrm{s}}{\tau_{i}} \geq 1.3 \times 10^{-3} .
$$

We first consider neutrino decay in the simple singlet model of sect. 2 . Assuming the neutrino mass is less than $5 \mathrm{meV}$, the constraint is

$$
\left(\frac{m_{\nu}}{\mathrm{MeV}}\right)^{2} \frac{\mathrm{GeV}}{v_{1}}\left(\frac{q_{2} v_{\mathrm{eff}}}{v_{1}}\right) \theta<5.8 \times 10^{-6}
$$

where $\theta=\sqrt{g_{\mathrm{a}, i j}^{2}+g_{\mathrm{v}, i j}^{2}}$ (see eq. (9)) is an off-diagonal coupling strength expected to be much less than one. If $\theta$ and $q_{2} v_{\text {eff }} / v_{1}$ were equal to one, the decay constraint is stronger than the scattering constraint for $v>14 \mathrm{GeV}$, but for $\theta=10^{-3}$ they are comparable at $v=140 \mathrm{GeV}$. For $m_{\nu}<30 \mathrm{eV}$ and $v>100 \mathrm{GeV}$ neither constraint is restrictive, nor will either constraint become more strict if we dilute the neutrino-majoron coupling by adding extra scalars.

With explicit symmetry breaking the majoron gets a mass and we must consider the reactions, $\chi \leftrightarrow \nu \nu$. These reactions are more interesting than neutrino decay 
since (a) they can proceed through the diagonal neutrino majoron coupling and (b) the majoron mass can be larger than the neutrino mass and this allows for a faster decay. As an example, consider our toy model; with $v_{1}=v_{2}=v, q_{1}=2, q_{2}=\frac{1}{2}$, and $g_{\text {grav }}=1$, the constraint is

$$
\left(\frac{\mathrm{m}_{v^{\prime}}}{\mathrm{MeV}}\right)^{2} \frac{l}{\mathrm{GeV}} \leq 51
$$

If we confine ourselves to $m_{\nu}<30 \mathrm{eV}$ then the constraint on $v$ is quite weak, $v<10^{10} \mathrm{GeV}$. This is a fictitious constraint - the majoron mass increases above 10 MeV for $v \approx 5 \times 10^{4}$, and for masses above this our analysis does not apply. In eq. (45) the dependence on $v$ arises due to the $v^{3 / 2}$ dependence of $m_{x}$. In other models this dependence will differ, and so will the constraints, but generally for neutrino masses below $30 \mathrm{eV}$ big-bang nucleosynthesis does not pose any serious constraints.

\section{Supernova constraints}

Since the SN 1987A neutrino pulse had its expected duration (O(10 s)), any scenario where the supernova has its energy drained more rapidly is ruled out. In this way it is possible [6] to put a constraint on the majoron luminosity. Assuming the $\rho$ particles are heavy, the largest contribution to the majoron luminosity will come from the processes $\nu \nu \rightarrow \chi \chi, \nu^{\prime} \rightarrow \nu \chi$, and $\nu \rightarrow \nu \chi$, where the last is matter induced, and is a consequence of the difference in energy between helicity states [34]. Choi and Santamaria [6] give the constraints from these processes as

$$
\begin{array}{ll}
\nu \nu \rightarrow \chi \chi: & \frac{m_{\nu}}{v_{1}} \frac{\mathrm{GeV}}{v_{1}} \leqslant 2 \times 10^{-8}, \\
\nu^{\prime} \rightarrow \nu \chi: & \frac{\tau_{\nu}}{\mathrm{s}} \frac{\mathrm{MeV}}{m_{\nu}} \gtrsim 3 \times 10^{-5}, \\
\nu \rightarrow \nu \chi: & \frac{m_{\nu}}{v_{1}} \leq 8 \times 10^{-7} .
\end{array}
$$

The scattering constraint (eq. (46)) is slightly stronger than that from nucleosynthesis (eq. (41)), whereas the off-diagonal inverse decay constraint (eq. (47)) is some two orders of magnitude weaker than the corresponding constraint from nucleosynthesis (eq. (43)), and the diagonal argument (eq. (48)) has no analog for the early Universe. As with the nucleosynthesis constraints, one of the supernova constraints are relevant if we confine ourselves to "reasonable" values, $m_{v}<30 \mathrm{eV}$ and $l>100 \mathrm{GeV}$. The constraints were based on requiring the majoron luminosity 
to be less than $3 \times 10^{53} \mathrm{erg} / \mathrm{s}$ for a 0.85 solar mass core, with a radius of $10 \mathrm{~km}$, at a temperature of $50 \mathrm{MeV}$. Although this condition is somewhat ad hoc, similar criteria in the study of axion emission have proven useful as a guide to more detailed numerical studies [35].

In the present context of a massive majoron we need to add inverse majoron decay as an additional production mechanism. Actually, inverse majoron decay already occurs in standard majoron models. The decay of a massless majoron into two positive helicity neutrinos can proceed as the analog to the helicity flipping neutrino decay discussed by Choi and Santamaria [6]. The phase space considerations are similar and the matrix element is the same, so we expect a similar constraint. If the gravitationally induced majoron mass is larger than the effective neutrino mass, then the phase space will be enhanced. We therefore estimate the constraint from inverse majoron decay by scaling to eq. (48),

$$
\nu \nu \rightarrow \chi: \quad \frac{m_{\nu}}{v_{1}} \leq 8 \times 10^{-7} \frac{m_{\chi}}{100 \mathrm{keV}},
$$

where the neutrino effective mass is of order $100 \mathrm{keV}$.

The supernova constraints do not apply if the majorons are so strongly coupled that they are trapped inside a "majoron sphere" which has a blackbody luminosity of $<10^{53} \mathrm{erg} / \mathrm{s}$. Choi and Santamaria estimate that the interaction $\nu \chi \rightarrow \nu \chi$ will trap the majorons if

$$
\frac{m_{\nu}}{\mathrm{MeV}}\left(\frac{\mathrm{GeV}}{v_{1}}\right)^{2} \geq 3 \times 10^{-3}
$$

A comparison with the constraints from nucleosynthesis (eq. (41)) shows that: if majorons are coupled strongly enough to be trapped, then they would be brought into equilibrium in the early Universe. Thus, trapping is not a viable way of evading the supernova constraint *. Rather, the supernova constraints are an extension of the nucleosynthesis constraint. This is a general result, the thermal conditions in the supernova are not so different than in the early Universe, but the time scale required for trapping is shorter than the expansion rate in the early Universe at comparable temperatures. For example to trap in the core requires an interaction time less than the light crossing time of $3 \times 10^{-5} \mathrm{~s}$, whereas the expansion time at $T=50 \mathrm{MeV}$ is $2 \times 10^{-4} \mathrm{~s}$. Actually, there is an even stronger requirement [37]: to avoid excess heat transport in the supernova core requires significantly larger cross sections than needed just for trapping, which would lead to even stronger disagreement with the nucleosynthesis constraint.

The main weakness, apart from numerical uncertainty, with the constraints summarized above is that they rely on the standard cold core-bounce model of

* For a possible exception to this, see ref. [36]. 
type-II supernovae being correct, but that scenario may not be consistent with the hypothesized majoron parameters. The crucial point is that if lepton number is strongly violated that the lepton degeneracy may be erased on infall. The resultant increase in entropy may cause a "thermal" bounce [38], and it is unknown whether or not such a scenario can produce the observed neutrino pulse from SN 1987A.

Other constraints may arise if particles produced in the supernova decay in flight to observable particles while on the way to Earth. In the majoron/neutrino sector, the most readily observable particle is the $\bar{\nu}_{\mathrm{e}}$. Two scenarios have been discussed. (a) Heavy neutrinos emitted from the neutrino sphere can decay producing a $10-20 \mathrm{MeV} \bar{\nu}_{\mathrm{e}}$ signal. Such a signal would have to be temporally separated from the primary neutrino pulse in order to be readily identifiable [39]. (b) High energy majorons from the core can decay producing a $\sim 100 \mathrm{MeV}$ signal [40]. Such a signal can be identified by its spectrum. Indeed, a single high energy event coincident with the primary neutrino burst would be significant. Since the neutrino detection cross sections increase with energy, only a fraction of the supernova energy must be emitted in high energy particles to produce a signal. The lack of any high energy events coincident with SN 1987A therefore places a constraint on the production processes for majorons that decay into $\bar{\nu}_{\mathrm{e}}$ 's which is $\sim$ one order of magnitude more stringent in the amplitude than the limits in eq. (46-48).

\section{Summary}

In this paper we argue that physics at the Planck scale may have interesting consequences for majoron models. Majoron models contain a global symmetry which, when broken, provides both for neutrino masses and relatively rapid neutrino decay; however, gravity need not respect global symmetries. Unless the global symmetry arises as an automatic consequence of a local symmetry, there is no reason to expect the symmetry to survive at all in the low energy phenomenology. To this end we have constructed explicit models where B-L is a spontaneously broken local gauge symmetry and the majoron arises as the Goldstone boson of a spontaneously broken "accidental" $U(1)$ symmetry. By varying the $\mathrm{B}-\mathrm{L}$ charge of a new scalar field in the model, the global symmetry can be protected against operators with dimension less than some power $d$. The most interesting cases are those where $d>4$, in which case the explicit breaking of lepton number is suppressed by $1 / M_{\mathrm{pl}}^{(d-4)}$.

The result of explicitly breaking $L$ is that the majoron gets a mass, which may have interesting consequences. In addition, by invoking an automatic symmetry to protect against explicit symmetry breaking the majoron is necessarily a linear composition of two scalar fields, only one of which couples to neutrinos. As a result, the majoron coupling to neutrinos may be suppressed from its value in simpler majoron models. 
By appropriate choices of parameters the majorons may be stable, and thermal relic majorons may play the role of dark matter. Alternatively, they may decay into light neutrinos with possible consequences for galaxy formation, or be so light and weakly coupled that they have almost no observable consequences at all. If the dimension of the gravitational operators is large enough, $(d \geqslant 10)$, there is the possibility of a coherent energy density in majorons, similar to that which arises in axion models.

If the majoron mass lies in the $\mathrm{MeV}$ region there will be consequences for big-bang nucleosynthesis. For lighter (keV) majorons, as long as neutrino masses lie below $30 \mathrm{eV}$ neither neutrino annihilation into majorons nor inverse majoron decay are likely to pose a problem for nucleosynthesis. Supernova constraints on majoron models are hardly altered by the majoron's mass, unless it exceeds a few hundred $\mathrm{MeV}$, which would suppress thermal majoron production in supernovae. Suppression of the majoron-neutrino coupling can soften the constraints from supernovae, even for light majorons.

Finally, there is not complete freedom in choosing the B-L charges for the scalar fields in the theory. An inappropriate choice implies the formation of a network of cosmological strings and domain walls that cannot dissipate via self-intersection. The energy density in such a network would be prohibitively large, and so such choices are proscribed.

D.S. and K.S.B. are partly supported by DOE grant DE-AC02-78ER05007. I.Z.R. is supported by NSF grants NSF-AST-9296246 and NSF-PHY-9296020. We acknowledge discussions on similar matters with R.N. Mohapatra. D.S. thanks S. Petcov, H. Haber and J. Cline for helpful discussions.

\section{References}

[1] Y. Chikashige, R.N. Mohapatra and R.D. Peccei, Phys. Lett. B98 (1981) 265

[2] G. Gelmini and M. Roncadelli, Phys. Lett. B99 (1981) 411;

H. Georgi, S. Glashow, and S. Nussinov, Nucl. Phys. B193 (1981) 297

[3] M. Gell-Mann, P. Ramond and R. Slansky, in Supergravity (North-Holland, Amsterdam, 1979); T. Yanagida, in Proc. Workshop on Unified theories and baryon number in the Universe; R.N. Mohapatra and G. Senjanovic, Phys. Rev. Lett. 44 (1980) 912

[4] For a review of standard cosmology, we refer to E.W. Kolb and M.S. Turner, The early Universe (Addison-Wesley, Reading, MA, 1990)

[5] For a review, see R.N. Mohapatra and P.B. Pal, Massive neutrinos in physics and astrophysics (World Scientific, Singapore, 1991)

[6] K. Choi and A. Santamaria, Phys. Rev. D42 (1990) 293;

K. Choi, C.W. Kim, J. Kim and W.P. Lam, Phys. Rev. D37 (1988) 3325;

J.A. Grifols, E. Masso and S. Peris, Phys. Lett. B215 (1988) 593

[7] S. Bertolini and G. Steigman, Ohio State preprint OSU-TA-2/92 (1992)

[8] D. Dearborn, D. Schramm and G. Steigman, Phys. Rev. Lett. 56 (1986) 26; G.G. Raffelt, Phys. Rev. D33 (1986) 897;

H.Y. Cheng, Phys. Rev. D36 (1987) 1649 
[9] For a recent review, see S.C.C. Ting, Plenary talk at 1992 DPF Meeting, Fermilab, November 1992

[10] For reviews, see F.T. Avignone and R.L. Brodzinski, Prog. Part. Nucl. Phys. 21 (1988) 99;

D. Caldwell, Nucl. Instrum. Meth. A264 (1988) 106

[11] For recent reviews, see J. Preskill, Caltech preprint CALT-68-1819 (1992);

T. Banks, Physicalia $12(1990) 19$

[12] R. Holman, S. Hsu, T. Kephart, E.W. Kolb, R. Watkins and L. Widrow, Phys. Lett. 282 (1992) 132;

M. Kamiokowski and J. March-Russell, Phys. Lett. 282 (1992) 137;

K.S. Babu and S.M. Barr, Bartol preprint BA092-79 (1992)

[13] S.M. Barr and D. Seckel, Phys. Rev. D46 (1992) 539

[14] J. Frieman and A. Jaffe, Phys. Rev. D45 (1992) 2674

[15] R. Holman et al., Phys. Rev. Lett. 69 (1992) 1489;

M. Komionkowski and J. March Russell, Phys. Rev. Lett. 69 (1992) 1485

[16] E.Kh. Akhmedov, Z. Berezhaini and G. Senjanovic, Phys. Rev. Lett. 69 (1992) 3013

[17] E.Kh. Akhmedov, Z.G. Berezhiani, R.N. Mohapatra and G. Senjanovic, Univ. of Maryland preprint, UMDHEP 93-020 (1992)

[18] J. Schecter and J.W.F. Valle, Phys. Rev. D25 (1982) 774

[19] G. Steigman and M.S. Turner, Nucl. Phys. B253 (1985) 375

[20] S.L. Glashow, Phys. Lett. B187 (1987) 367

[21] P.B. Pal, in Beyond the Standard Model II, eds. K. Milton, R. Kantowski and M. Samuel (World Scientific, Singapore, 1991)

[22] K.S. Babu and R.N. Mohapatra, Phys. Rev. D46 (1992) 374;

A.S. Joshipura and S. Rindani, Ahmedabad preprint (1992)

[23] K.S. Babu, R.N. Mohapatra and I.Z. Rothstein, Phys. Rev. Lett. 67 (1991) 545

[24] X-He, G.C. Joshi, H. Lew and R.R. Volkas, Phys. Rev. D44 (1991) 2118

[25] L. Wolfenstein, Phys. Rev. D17 (1978) 2369; S.P. Mikheyev and A.Yu. Smirnov, Yad. Fix. 42 (1985) 1441

[26] J. Preskill, M. Wise and F. Wilczek, Phys. Lett. B120 (1983) 127; L. Abbott and S. Sikivie, Phys. Lett. B120 (1983) 133;

M. Dine and W. Fischler, Phys. Lett. B120 (1983) 137

[27] R.L. Davis and P. Shellard, Nucl. Phys. B234 (1989) 167

[28] D. Harari and P. Sikivie, Phys. Lett. B195 (1987) 361

[29] A. Vilenkin and A.E. Everett, Phys. Rev. Lett. 48 (1982) 1867

[30] R.L. Davis, Phys. Lett. B161 (1985) 285;

D.P. Bennett, Phys. Rev. D34 (1986) 3592

[31] T.P. Walker, G. Steigman, D. Schramm, K.A. Olive and H.-S. Kang, Astrophys. J. 376 (1991) 51

[32] K.S. Babu and I.Z. Rothstein, Phys. Lett. B275 (1992) 112

[33] S. Dodelson and M.S. Turner, Fermilab preprint Fermilab-Pub-92/84-A (1992)

[34] Z.G. Berezhiani and M.I. Vysotsky, Phys. Lett. B199 (1987) 281;

Z.G. Berezhiani and A.Yu. Smirnov, Phys. Lett. B220 (1989) 279

[35] A. Burrows, M.S. Turner and R. Brinkman, Phys. Rev. Lett. 39 (1989) 1020

[36] K.S. Babu, R.N. Mohapatra and I.Z. Rothstein, Phys. Rev. D45 (1992) R3312

[37] G. Raffelt and D. Seckel, in preparation

[38] G. Fuller, R. Mayle and J. Wilson, Astrophys. J. 332 (1988) 826

[39] J. Frieman, H. Haber and K. Freese, Phys. Lett. B200 (1988) 115; J.M. Soares and L. Wolfenstein, Phys. Rev. D40 (1989) 3666;

G. Geimini, S. Nussinov and R.D. Peccei, Intern. J. Mod. Phys. A7 (1992) 3141;

R.N. Mohapatra and S. Nussinov, Maryland preprint (1991)

[40] S. Dodelson, J. Frieman and M.S. Turner, Phys. Rev. Lett. 68 (1992) 2572 